$\operatorname{IPPP} / 08 / 94$

DCPT $/ 08 / 188$

LTH 816

29 May 2009

\title{
Generalised parton distributions at small $x$
}

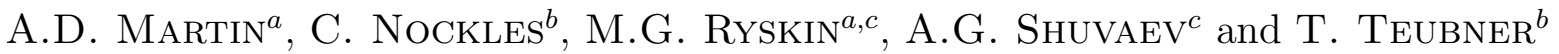 \\ ${ }^{a}$ Department of Physics and Institute for Particle Physics Phenomenology, \\ University of Durham, Durham DH1 3LE, U.K. \\ ${ }^{b}$ Department of Mathematical Sciences, \\ University of Liverpool, Liverpool L69 3BX, U.K. \\ ${ }^{c}$ Petersburg Nuclear Physics Institute, Gatchina, St. Petersburg, 188300, Russia
}

\begin{abstract}
We justify the practical use of the Shuvaev integral transform approach to calculate the skewed distributions, needed to describe diffractive processes, directly from the conventional diagonal global parton distributions. We address doubts which have been raised about this procedure. We emphasise that the approach, on the one hand, satisfies all theoretical reqirements, and, on the other hand, is consistent with DVCS data at NLO. We construct an easily accessible package for the computation of these skewed distributions.
\end{abstract}

\section{Motivation}

Skewed parton distributions are needed to calculate exclusive diffractive production, such as $\gamma+p \rightarrow V M+p$ for light or heavy vector mesons, see e.g. [1, 2, 3, 4, central exclusive diffractive Higgs boson production at the LHC [5], etc. For all these diffractive processes we need skewed parton distributions at small values of $\xi \ll 1$, and small to medium scales. Data for diffractive $J / \psi$ production, for example, test the gluon in the range $\xi \simeq x=10^{-4} \ldots 5 \cdot 10^{-3}$ 


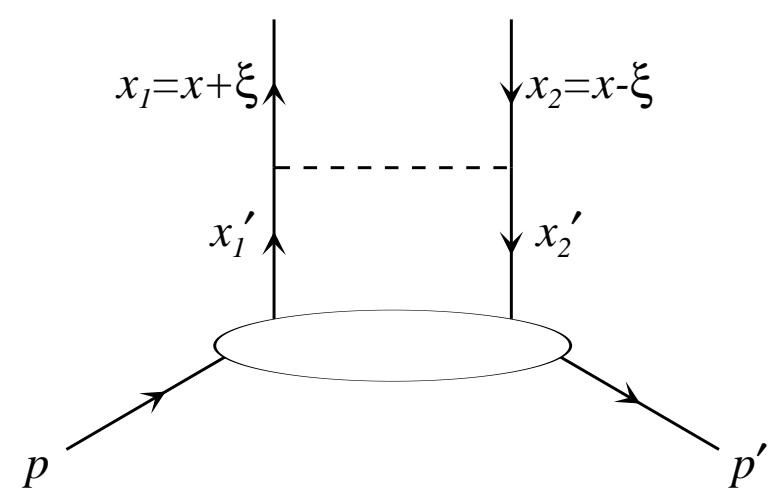

Figure 1: A schematic diagram showing the variables for the off-diagonal parton distribution $H(x, \xi)$ where $x_{1,2}=x \pm \xi$.

and effective scales $\mu^{2} \sim 2 \ldots 8 \mathrm{GeV}^{2}[6]$. For exclusive Higgs production at the LHC, the regime of $\xi \simeq x \simeq 10^{-2}$ and scales of the order $5 \mathrm{GeV}^{2}$ will be relevant, see [5].

Moreover it has been proved, using dispersion relations [7], that only distributions in the space-like region $|x|>\xi$ are needed to describe these processes. The variables are defined in Fig. 1.

There are insufficient experimental data to determine the skewed parton distributions with an accuracy comparable to that of the global parton analysis of the conventional (diagonal) distributions. Fortunately, for small $\xi$, the skewed distributions can be computed accurately just from the knowledge of the known integrated conventional distributions. At first sight such a simplification looks surprising. On the other hand, we know that the anomalous dimensions which describe the evolution of the Gegenbauer moments, $G_{N}$, of the skewed distributions are equal to the corresponding anomalous dimensions of the conventional Mellin moments, $M_{N}$ [8, 9]. This is a consequence of conformal invariance of the evolution equations. Strictly speaking, conformal invariance is only valid at leading order (LO). Already at NLO [10, 11], it is violated by the running of $\alpha_{S}$, leading to a mixture of the operators at adjacent orders. But let us start with LO.

At LO we have equality of the anomalous dimensions of $G_{N}$ and $M_{N}$. Moreover, due to the polynomial property [12, 13, 14],

$$
G_{N}=\sum_{n=0}^{N} c_{n}^{N} \xi^{2 n},
$$

we have $c_{0}^{N}=M_{N}$. That is, from the conventional global parton analyses we can determine all the Gegenbauer moments of the skewed parton distributions at small $\xi$ with an accuracy of $O\left(\xi^{2}\right)$. Then it is simply a technical problem to calculate the $x$ distribution of the skewed 
partons from the known moments. This mathematical problem is solved by the Shuvaev transform [15] (which we give explicitly later). Note that at $\xi=0$, in the diagonal case, there is no mixture of the Mellin moments during the evolution. Thus for the skewed distributions such mixing must also vanish at $\xi=0$. Indeed the mixture of the different operators, due to violation of conformal invariance 1 , is of $O\left(\alpha_{s} \xi\right)$ [10, 11, 16, 17]. Thus the Shuvaev transform can be used at NLO with accuracy $O(\xi)$, which is sufficient for the description of all diffractive processes of interest.

This procedure has been called into doubt [18]. First, we discuss the reason for the doubt and then explain why it is not a problem in practice. The apparent problem is that to obtain the $x$ distributions from the moments $G_{N}$ we must analytically continue the moments into the complex $N$-plane. If there is a singularity in the right-half plane, then it may generate a nonnegligible correction of $O(\xi / x)$, instead of the $O\left(\xi^{2}\right)$ correction which came from the difference between the Gegenbauer and Mellin moments. This large $O(\xi / x)$ correction would destroy the practical use of the Shuvaev transform. So to justify the Shuvaev transform we require the absence of singularities in the right-half $N$ plane.

Now, an arbitrary singularity in the right-half plane will violate the polynomial property, and so at first sight the danger is removed. However, it has been shown by Radyushkin [19, 20, 21, 22] that it is possible to form 'double distributions' which are not identical to the Shuvaev transform, but which still satisfy the polynomial property. The non-polynomial contributions, generated by different singularities of these double distributions in the right-half plane, compensate each other to guarantee polynomiality. So the danger remains. On the other hand, there is no singularity in the right-half plane in the anomalous dimensions which describe the $q^{2}$ evolution of the Gegenbauer moments. Hence the extra singularities must come only from the input distribution. Now, it is natural to describe the input distribution at low $x$ in terms of the Regge approach, which is successful in the description of high energy interactions at low scales where the conventional (collinear) DGLAP evolution for the skewed distributions starts. In the Regge approach there are no singularities in the right-half plane $(j>1)$ in the space-like $(x>\xi)$ domain.

Indeed, in the Regge limit we are concerned with the leading $\log (1 / x)$ summation. Hence, in Fig. 2 we have the strong ordering

$$
x_{i} \gg x_{i+1}^{\prime}, \quad \text { that is }\left|x_{i}-x_{i}^{\prime}\right| \equiv 2 \xi \ll x_{i} \quad \text { for } \quad i<n \text {. }
$$

The inequality $\xi \ll x_{i}$ means that the lower part of the diagram is described by the diagonal distribution. The only $\xi$ dependence comes from the uppermost $x_{n}$ cell. All other cells have $x_{i} \gg \xi$. Now, the uppermost cell already satisfies conformal invariance. Moreover, at NLO level, this uppermost cell is calculated exactly in terms of the NLO coefficient functions. Therefore there is no opportunity for a new singularity in the right-half $j>1$ plane.

\footnotetext{
${ }^{1}$ This violation occurs due to the dependence of $\alpha_{s}$ on the dimensionful parameter $\Lambda_{\mathrm{QCD}}$. It is essentially trivial. In principle, it could be accounted for by using the 'correct' argument of the QCD coupling in the uppermost cell of Fig. 1] namely $\alpha_{s}\left(k_{t}^{2}\right)$ and not $\alpha_{s}\left(Q^{2}\right)$.
} 


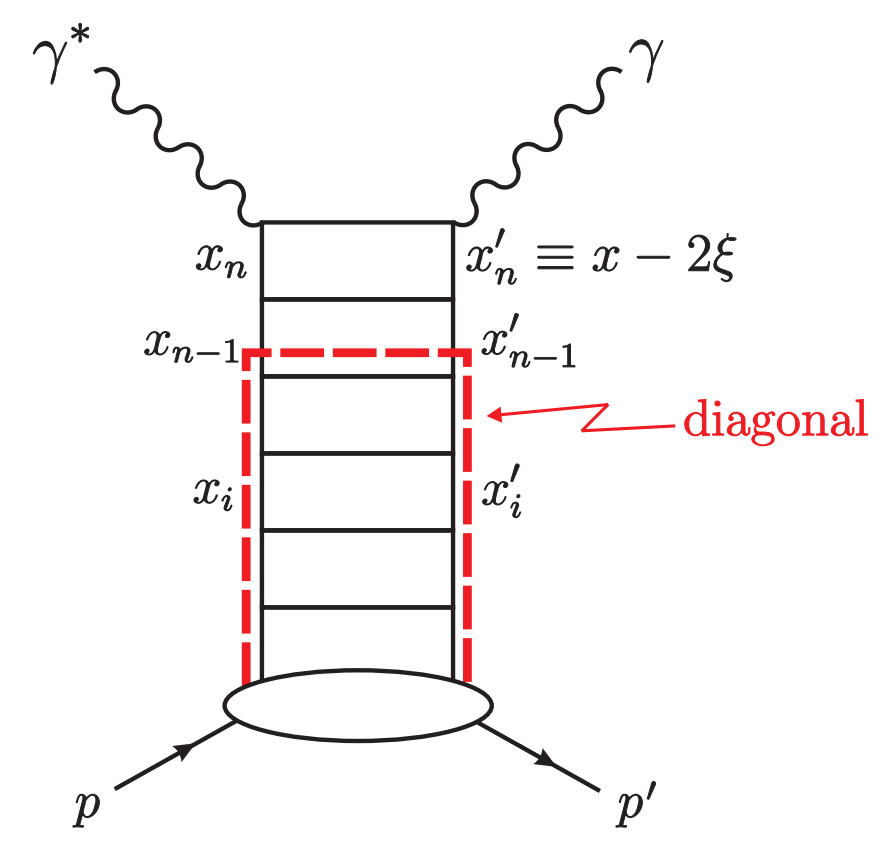

Figure 2: Schematic diagram describing deeply virtual Compton scattering (DVCS) in terms of generalised parton distributions. The skewing (or $\xi$ dependence) originates from the uppermost cell; the part of the diagram enclosed by the dashed line has diagonal form due to the strong ordering of $x$ in the Regge limit. This diagram makes clear why, in the low $x$ region, generalised parton distributions may be computed directly from the well known 'global' diagonal distributions.

With the above physically motivated conjecture, the Shuvaev transform survives to be of practical value. We emphasise that the conjecture, of the absence of additional singularities in the right-half plane, does not follow from first principles or symmetry arguments, but rather from the assumption of the Regge form of the low $x$ input.

Note that in the time-like region, that is at $|x|<\xi$, we have such singularities. They correspond to the wave functions of resonances with large spin. Indeed one may add to the distribution, given by the Shuvaev transform, an additional function with support only on the interval $|x|<\xi$. However there is no such contribution in the calculation of the skewed partons via the Shuvaev transform in the space-like domain 2

Another argument in favour of the Shuvaev prescription is the good fit of the DVCS HERA data obtained at NLO and NNLO in [23]. This fit was based on the approximation that there is only one pole. Recall also the model [24] based on the Shuvaev transform and the successful description of the DVCS HERA data in the dipole model [25] where the ratio, $R_{g}$, of the skewed to diagonal gluon distribution given by the Shuvaev transform was used.

\footnotetext{
${ }^{2}$ As was pointed out in [24, such 'meson-wave-function-like' contributions lead to extra terms in the inverse Shuvaev transform, which we do not use here. Also note that similar extra terms in the effective diagonal functions as proposed in 24] are irrelevant for our case.
} 
In [26] the Shuvaev transform was criticised to be "unpractical" or "too complicated", but see also the original discussion in [27]. Here we present and describe a package that allows for the simple computation of the skewed distributions at small $x, \xi$ based on the Shuvaev transform.

\section{Description of the Shuvaev transform}

The generalised parton distributions (GPDs) are denoted [12, 13, 14] by $H_{i}(x, \xi), i=q, g$ where $\xi$ is the skewing parameter, with $x_{1,2}=x \pm \xi$ as defined in Fig. 1. In fact $H(x, \xi) \equiv H\left(x, \xi, t, \mu^{2}\right)$ for partons emitted and absorbed at scale $\mu^{2}$, with the momentum transfer $t=\left(p-p^{\prime}\right)^{2}$, and $-1<x<1$. The values of $t$ and $\xi$ do not change as we evolve in $\mu^{2}$. In the limit $\xi \rightarrow 0$ the skewed distributions will reduce to the usual diagonal partons:

$$
\begin{aligned}
& H_{q}(x, 0)=\left\{\begin{array}{ll}
q(x) & \text { for } \quad x>0 \\
-\bar{q}(-x) & \text { for } \quad x<0
\end{array},\right. \\
& H_{g}(x, 0)=x g(x) .
\end{aligned}
$$

We briefly discuss the double-integral (that is, the Shuvaev transform) used to calculate the skewed distributions for $\xi \ll 1$ in the space-like region $|x|>\xi$. First we introduce an auxiliary function $f_{\xi}(x, t)$, whose Mellin moments are equal up to normalisation to the Gegenbauer moments of the skewed function. The function formed from the DGLAP evolution of the auxiliary function is referred to as the 'effective diagonal function', $f\left(x^{\prime}\right)$. Neglecting the $t$ dependence, we must find the kernel $K\left(x, \xi ; x^{\prime}\right)$ relating $f\left(x^{\prime}\right)$ to the skewed distribution,

$$
H(x, \xi)=\int \mathrm{d} x^{\prime} K\left(x, \xi ; x^{\prime}\right) f\left(x^{\prime}\right) .
$$

It is convenient to first weaken the singularity in the $x^{\prime}$ integral by an integration by parts. With this it can be shown that [28]

$$
\begin{gathered}
H_{q}(x, \xi)=\int_{-1}^{1} \mathrm{~d} x^{\prime}\left[\frac{2}{\pi} \operatorname{Im} \int_{0}^{1} \frac{\mathrm{d} s}{y(s) \sqrt{1-y(s) x^{\prime}}}\right] \frac{\mathrm{d}}{\mathrm{d} x^{\prime}}\left(\frac{q\left(x^{\prime}\right)}{\left|x^{\prime}\right|}\right) \\
H_{g}(x, \xi)=\int_{-1}^{1} \mathrm{~d} x^{\prime}\left[\frac{2}{\pi} \operatorname{Im} \int_{0}^{1} \frac{\mathrm{d} s(x+\xi(1-2 s))}{y(s) \sqrt{1-y(s) x^{\prime}}}\right] \frac{\mathrm{d}}{\mathrm{d} x^{\prime}}\left(\frac{g\left(x^{\prime}\right)}{\left|x^{\prime}\right|}\right), \\
y(s)=\frac{4 s(1-s)}{x+\xi(1-2 s)} .
\end{gathered}
$$

Equations (5) and (6) are (up to a variable substitution $z=1 /\left(x^{\prime} y(s)\right)$ ) the form used to compute the Shuvaev transform in the package decribed below. 
Incidentally, it is also possible to solve the inverse problem. That is to obtain the diagonal distribution from a known skewed distribution $H(x, \xi)$ at a given value of $\xi$. Although this is not needed for our discussion, for completeness, we give the details in the Appendix.

Note that the transforms, (5) and (6), say nothing about the $t$ (or $p_{T}$ ) dependence of the GPDs. Strictly speaking they are written for $p_{T}=0$. Usually the factorisation

$$
H\left(x, \xi ; p_{T}\right)=H(x, \xi) F\left(p_{T}\right)
$$

is assumed, where $F\left(p_{T}\right)$ is just the proton form factor.

\section{Approximate predictions of GPDs for small $x$ and $\xi$}

We see that (15) and (6) determine the behaviour of the skewed distributions in the small $x, \xi$ domain entirely in terms of the diagonal distributions. Before we perform the exact evaluation of these integral expressions for the GPDs, it is informative to recall that approximate expressions can be obtained by making the physically reasonable small $x$ assumption that the diagonal partons are given by

$$
x q(x)=N_{q} x^{-\lambda_{q}}, \quad x g(x)=N_{g} x^{-\lambda_{g}} .
$$

Then we can perform the $x^{\prime}$ integration analytically 3 We obtain

$$
H_{i}(x, \xi ; t)=N_{i} \frac{\Gamma\left(\lambda+\frac{5}{2}\right)}{\Gamma(\lambda+2)} \frac{2}{\sqrt{\pi}} \int_{0}^{1} \mathrm{~d} s[x+\xi(1-2 s)]^{p}\left[\frac{4 s(1-s)}{x+\xi(1-2 s)}\right]^{\lambda_{i}+1} G(t)
$$

with $i=q$ or $g$, and where $p=0$ and 1 for quarks and the gluon respectively.

At first sight it appears that for singlet quarks (where $\lambda_{q}>0$ and $p=0$ ) we face a strong singularity in integral (10) when the term $D \equiv x+\xi(1-2 s) \rightarrow 0$ in the denominator. Fortunately the singlet quark distribution is antisymmetric in $x$. To obtain the imaginary part of the integral (5) we must choose $x^{\prime}>0$ for $D>0$ and $x^{\prime}<0$ for $D<0$. Therefore we must treat (10) as a principal value integral and take the difference between the $D \rightarrow 0+$ and $D \rightarrow 0$ - limits. Thus the main singularity is cancelled and (10) becomes integrable for any $\lambda_{q}<1$.

Note that the dominant contribution to the $x^{\prime}$ integrations of (5) and (6) comes from the region of small $x^{\prime} \sim x, \xi$. Indeed with the input given by (9), the integral for the quark

\footnotetext{
${ }^{3}$ With the substitution $z=1 / x^{\prime} y(s)$ one can then use

$$
\int_{0}^{1} \mathrm{~d} z z^{\lambda+\frac{3}{2}}(1-z)^{-\frac{1}{2}}=\frac{\Gamma\left(\lambda+\frac{5}{2}\right) \Gamma\left(\frac{1}{2}\right)}{\Gamma(\lambda+3)},
$$

where we have set the lower limit to zero. We have checked numerically that this is a very good approximation, with accuracy of the order of $10^{-4}$ for $\lambda \simeq 0.2$ in the small $x$ region. (That this approximation is good for small $\xi$ is in line with the findings of 24].)
} 


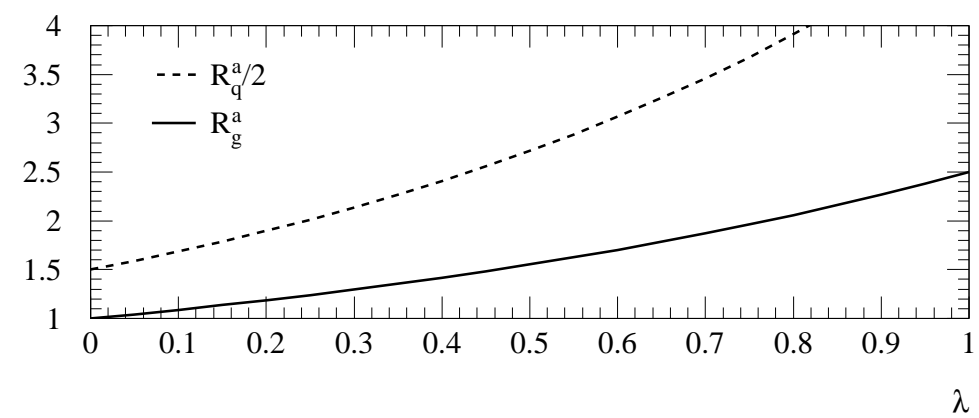

Figure 3: The off-diagonal to diagonal ratio, $R^{a}$, at small $x=\xi$ versus the power $\lambda$ which specifies the $x^{-\lambda}$ behaviour of the input diagonal parton as in (9). Note that the quark singlet ratio has been divided by two.

distribution has a strong singularity at small $x^{\prime}$

$$
I_{q} \sim \int \mathrm{d} x^{\prime}\left(x^{\prime}\right)^{-\lambda_{q}-3} \operatorname{Im}\left(\frac{1}{y(s) \sqrt{1-y(s) x^{\prime}}}\right) .
$$

However when we take the imaginary part, the $x^{\prime}$ integration is cut-off by the theta function $\theta\left(x^{\prime}-1 / y(s)\right)$ at

$$
x^{\prime}=1 / y(s) \sim x+\xi(1-2 s),
$$

which implies that $x^{\prime}$ is always greater than $x / 2$. So we obtain the small $\xi$ behaviour $I_{q} \sim \xi^{-\lambda_{q}-1}$, and the distribution (5) has the form

$$
H_{q}(x, \xi)=\xi^{-\lambda_{q}-1} F_{q}(x / \xi) .
$$

Similarly it follows that $H_{g}=\xi^{-\lambda_{g}} F_{g}(x / \xi)$.

It is illuminating to evaluate the ratio $R$ of the GPD to its diagonal parent distribution; that is

$$
R=\frac{H(x, \xi)}{H(x+\xi, 0)},
$$

where the only free parameter is $\lambda$, the exponent which fixes the $x^{-\lambda}$ behaviour of the input diagonal partons, as in (9). Notice that on account of (13) the ratios $R$ at small $x$ and $\xi$ are a function of only the ratio of the variables $x / \xi$.

Assuming the pure power behaviour of the partons, the ratios at $x=\xi$ are given explicitly in analytic form as

$$
R^{a}=\frac{H(\xi, \xi)}{H(2 \xi, 0)}=\frac{2^{2 \lambda+3}}{\sqrt{\pi}} \frac{\Gamma(\lambda+5 / 2)}{\Gamma(\lambda+3+p)},
$$

where $p=0$ for quarks and $p=1$ for gluons. These ratios are plotted in Fig. 3 as a function of $\lambda$. We see that the off-diagonal or 'skewed' effect (the ratio $R^{a}$ ) is much stronger for singlet quarks 
than for gluons. The explanation is straightforward. At low $x$ the distributions are driven by the double leading logarithmic evolution of the gluon distribution. At each step of the evolution the momentum fractions $x_{i}$ are strongly ordered $\left(x_{1}^{\prime} \gg x_{1}, x_{2}^{\prime} \gg x_{2}\right.$ on Fig. 1). For gluons it is just the "last splitting function" $P_{g g}\left(x_{2}, x_{2}^{\prime} ; \xi\right)$ which generates the main $\xi$ dependence, or skewedness, of the distribution. However for the sea or singlet quarks it is necessary to produce a quark with the help of $P_{q g}$ at the last splitting. The splitting function $P_{q g}$ has no logarithmic $1 / z=x_{2}^{\prime} / x_{2}$ singularity and so $x_{2}$ is the order of $x_{2}^{\prime}$. Consequently both the splitting functions $P_{q g}\left(x_{2}, x_{2}^{\prime} ; \xi\right)$ and $P_{g g}\left(x_{2}^{\prime}, x_{2}^{\prime \prime} ; \xi\right)$ generate the asymmetry of the off-diagonal distribution. Hence, at low $x$, the singlet quark has a much stronger off-diagonal effect than the gluon.

We emphasise that the analytical expression for the ratio $R^{a}$, (15), is valid in the limit $x=\xi$ only and that it assumes a pure power behaviour of the diagonal partons, (9). As will be discussed below, the latter is a good approximation for global fit partons in the small $x$ regime. However, for $x>\xi$ the difference between the result of the complete (double integral) Shuvaev transform, (5, 6), and the approximate analytic formula for $R^{a}$, (15), based on the limit $x=\xi$, is quite large. This is evident from Fig. 6 below.

\section{Evaluation of the GPDs using the Shuvaev transform}

In the above section we have obtained an approximate determination of the GPDs valid for $x \simeq \xi$. We now perform a precise evaluation of the Shuvaev transforms of (5) and (6), valid for all small $x, \xi$, and compare our results to the approximation based on (10). This is done in Fig. 4 for the gluon and in Fig. 5 for the sums of $u, d, s$ quarks and antiquarks, using MSTW2008NLO [29] (conventional global fit) parton distributions as input. Thus our GPDs correspond to using the $\overline{\mathrm{MS}}$ renormalisation scheme and the General Mass Variable Flavour Number Scheme adopted by MSTW.

In the lower panels of the figures the effective powers $\lambda$ are plotted as a function of $x$ for three different scales, $\mu^{2}=2.5,10,50 \mathrm{GeV}^{2}$. In the upper panels both the ratios

$$
\tilde{R}=\frac{H(x / 2, x / 2)}{H(x, 0)}
$$

for the full Shuvaev transform and the corresponding analytical approximations

$$
\tilde{R}^{a}=\frac{H(x / 2, x / 2)}{H(x, 0)}=\frac{2^{2 \lambda+3}}{\sqrt{\pi}} \frac{\Gamma(\lambda+5 / 2)}{\Gamma(\lambda+3+p)} \quad(\text { with } \lambda \text { evaluated at } x)
$$

are given, again for the three scales. It is clear that, depending on the values of $x$ and the scale, the deviation from a pure power can lead to a sizeable difference between the analytic approximation and the full result. However, for small $x \lesssim 2 \cdot 10^{-3}$ and not too small scales the deviation is quite small in the case of the global fit partons MSTW2008NLO. Actually the difference is smaller than it appears at first sight in Fig. 5 since the vertical scale does not extend to zero. 

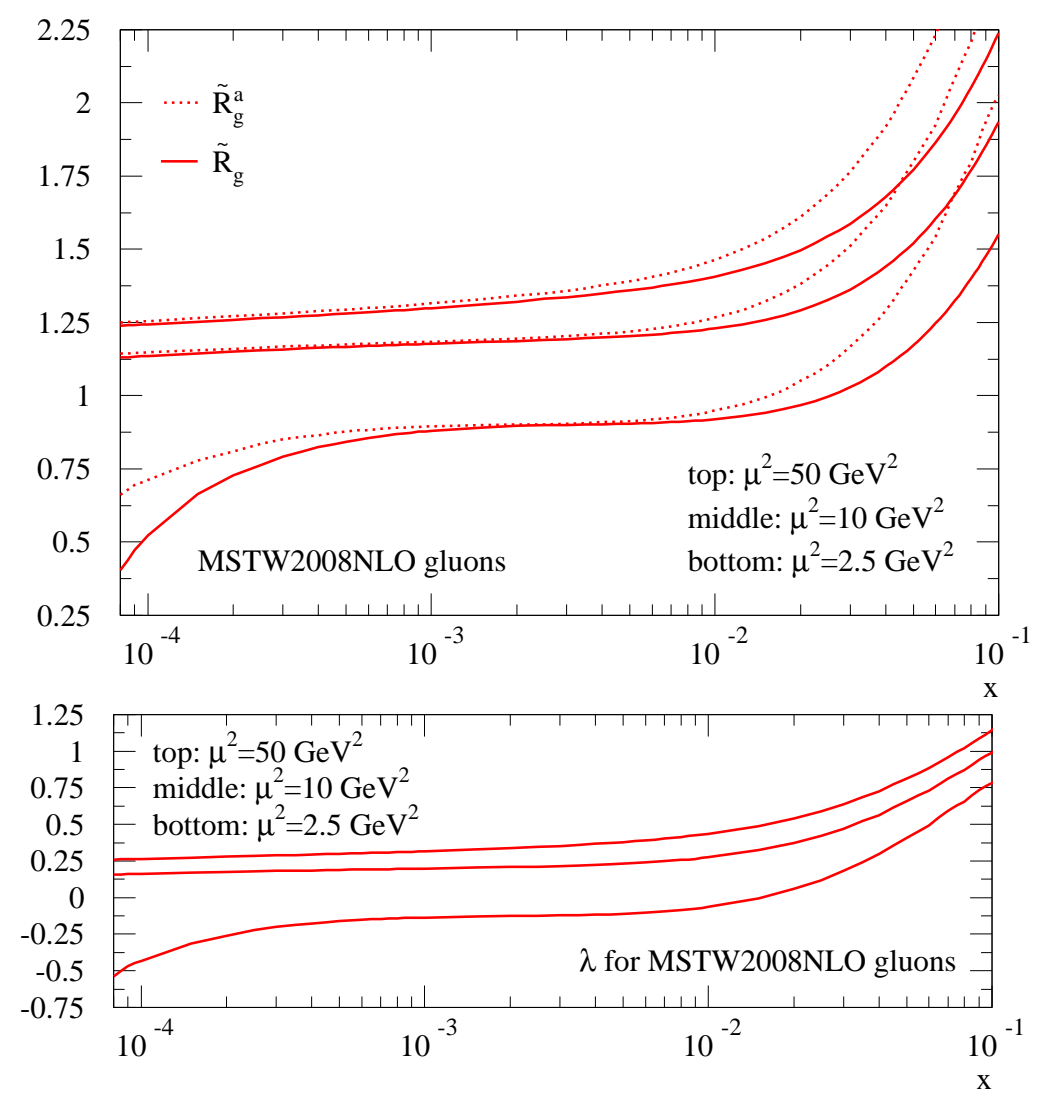

Figure 4: Lower panel: Values for the effective power $\lambda$ (evaluated at $x$ ) of the MSTW2008NLO gluon for the three scales $\mu^{2}=2.5,10,50 \mathrm{GeV}^{2}$. Upper panel: Analytic approximation $\tilde{R}_{g}^{a}$ (dotted lines) as defined in (17) compared to the ratio $\tilde{R}_{g}$ (solid lines) as defined in (16)) for the full Shuvaev transform. 

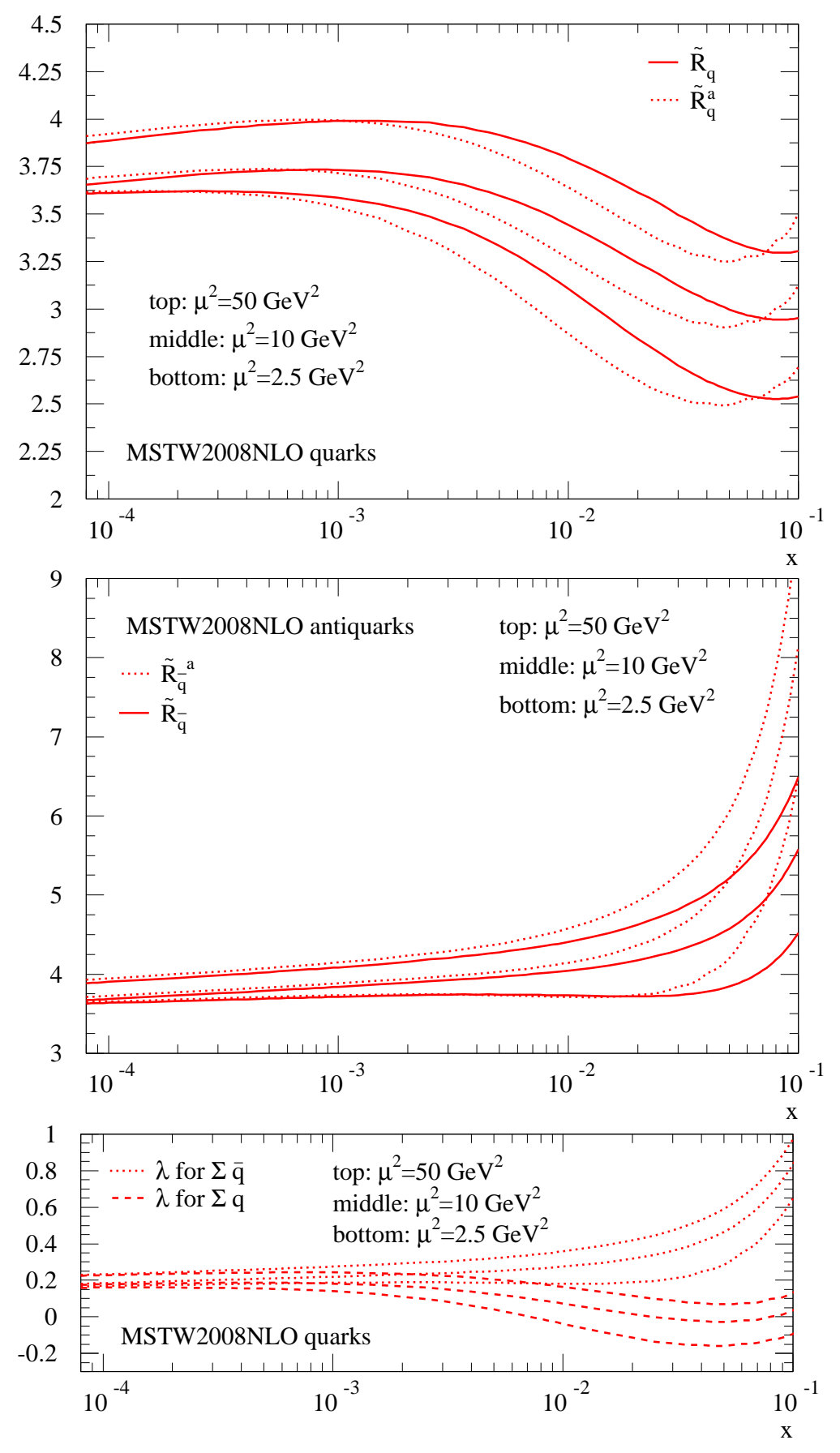

Figure 5: Lower panel: Values for the effective power $\lambda$ (evaluated at $x$ ) for the sum of the MSTW2008NLO $u, d, s$ quarks (dashed) and antiquarks (dotted lines) for the three scales $\mu^{2}=$ 2.5, 10, $50 \mathrm{GeV}^{2}$. Upper two panels: $\tilde{R}_{q}^{a}$ as defined in (17) shown as dotted lines for quarks (top panel) and antiquarks (middle panel) compared to the ratio $\tilde{R}_{q}$ for the full Shuvaev transform as defined from (16)) (solid lines). 

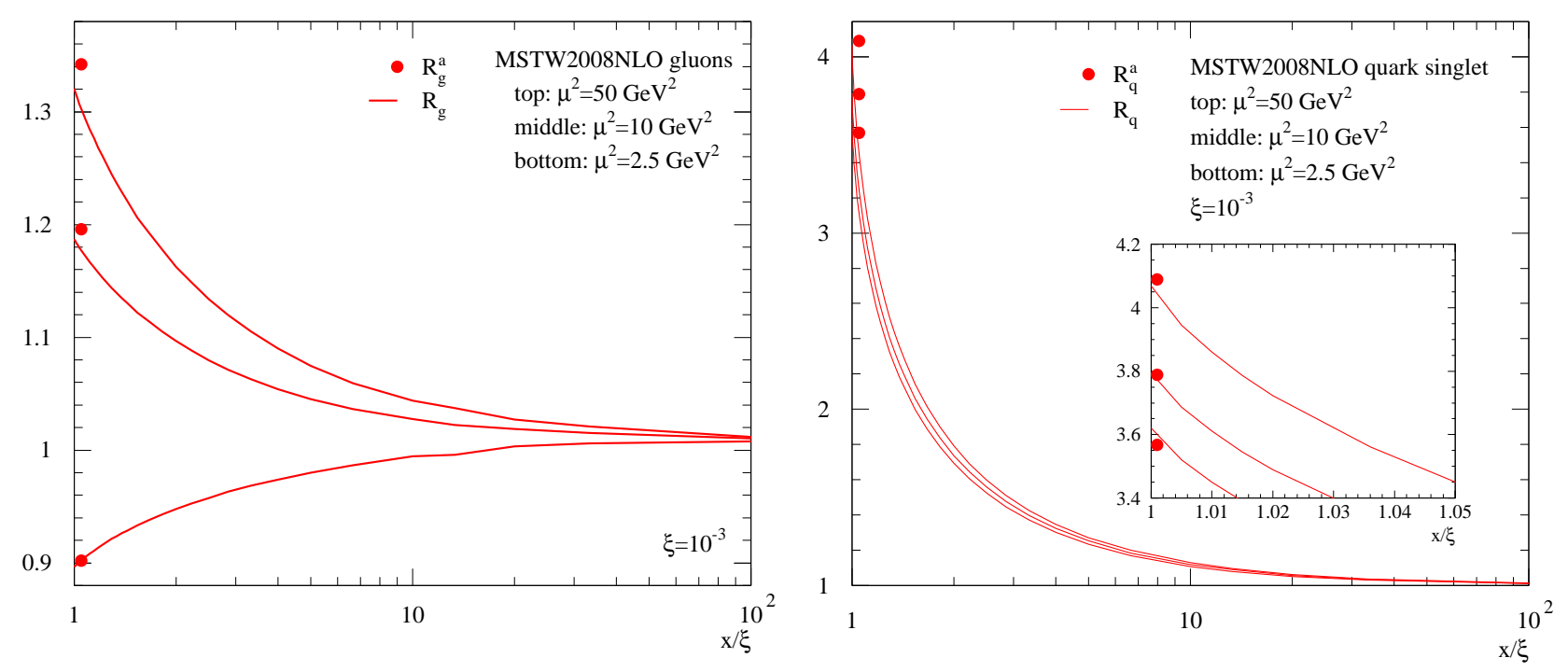

Figure 6: Left panel: Analytic $R_{g}^{a}$ from (15) (fat dots) compared to the ratio $R_{g}$ from (14) (solid lines) using the full Shuvaev transform ([6), at the three scales $\mu^{2}=2.5,10,50 \mathrm{GeV}^{2}$ for the MSTW2008NLO gluon and $\xi=10^{-3}$ as a function of $x / \xi$. Right panel: same as left panel but for the sum of $u, d, s$ quarks and antiquarks. The insert shows a blow-up of the regime $x \simeq \xi$.
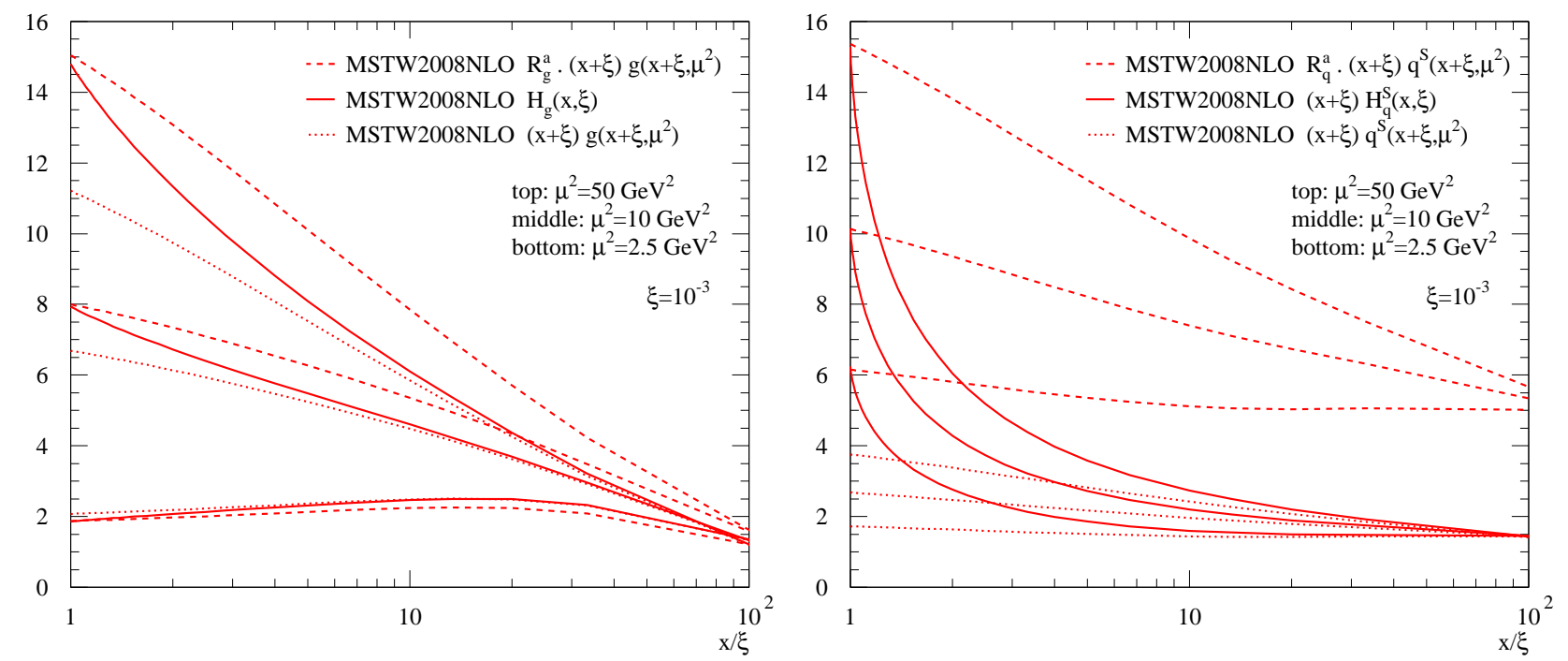

Figure 7: Comparison of skewed (solid) and diagonal (dotted lines) gluon (left) and singlet quark (right panel) MSTW2008NLO distributions for $\xi=10^{-3}$ and the three scales $\mu^{2}=$ $2.5,10,50 \mathrm{GeV}^{2}$ as a function of $x / \xi$. Also shown is the product of the diagonal distributions with the analytical skewing enhancement factor $R^{a}$ obtained in the $x=\xi$ limit from (15) (dashed lines). 

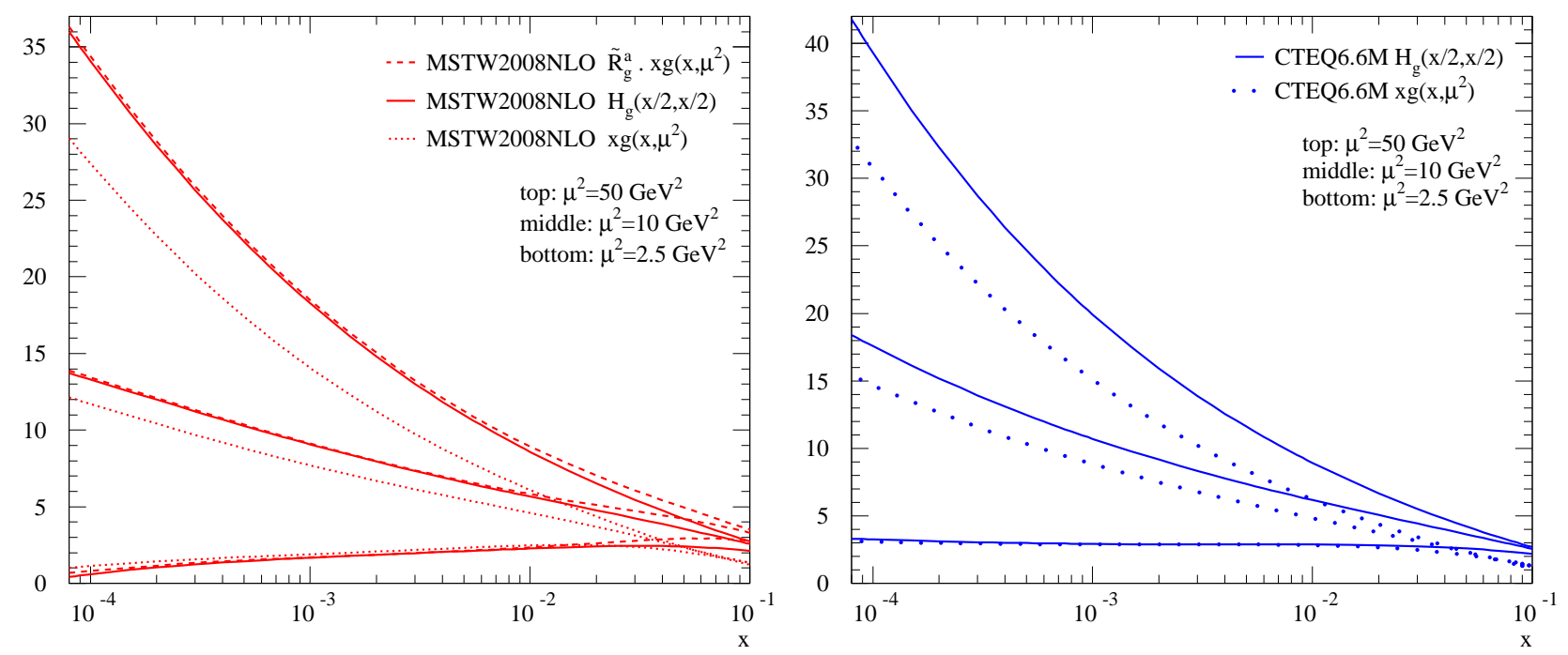

Figure 8: Skewed (solid and dash-dotted) and diagonal (dotted lines) MSTW2008NLO (left) and CTEQ6.6M (right panel) gluon distributions at scales $\mu^{2}=2.5,10,50 \mathrm{GeV}^{2}$. The dashed lines show the skewed gluon if the analytic approximation $\tilde{R}^{a}$ from (17) is used (MSTW only).

Frequently, skewing corrections are taken into account via simple multiplication with the ratio $R^{a}$. One may fear that this approximation of 'maximal skewing' overestimates the real skewing effect which comes from integrating over the whole possible range $\xi \leq|x|$. Possible effects are exemplified in Fig. 6 for MSTW2008NLO gluons (left) and the sum of $u, d, s$ quarks and antiquarks (right panel). Here the limit $R^{a}$ from (15) (fat dots) is compared to the full ratio $R$ from (14) (solid lines) as a function of $x / \xi$ for $\xi=10^{-3}$ at three different scales, $\mu^{2}=2.5,10,50 \mathrm{GeV}^{2}$.

In Fig. 7 a similar comparison is shown for the skewed gluon (left) and singlet quark distributions (right panel) as a function of $x / \xi$ for $\xi=10^{-3}$ at the three scales. The solid lines show the results for the full Shuvaev transforms, $H_{g}(x, \xi)$ and $(x+\xi) H_{q}^{s}(x, \xi)$, whereas the dashed lines are obtained as the product of the analytical skewing factors $R^{a}$ (in the limit $x=\xi$ ) with the diagonal partons evaluated at $x+\xi$. The dotted lines show the diagonal MSTW2008NLO partons for comparison. It is clear from Figs. [6 and 7 that, at least in the cases under consideration, the overestimate of skewing effects through simple multiplication with $R^{a}$ could be sizeable, although as expected the agreement when $x=\xi$ is good.

In Figs. 8 and 9 we finally show the skewed gluon, $H_{g}(x / 2, x / 2)$, and singlet quark distributions, $x H_{q}^{S}(x / 2, x / 2)$ (solid lines), compared to the corresponding diagonal partons (dotted lines) as a function of $x$ and at three scales for both MSTW2008NLO [29] and CTEQ6.6M [30]. For MSTW, the analytical approximation for the skewing using the factor $\tilde{R}^{a}$ is also shown (dashed lines).

Some features of these results are especially noteworthy. First, we emphasise that for $x \gg \xi$ the skewed distribution $H(x, \xi)$ becomes close to the diagonal distribution. This is trivial, since 


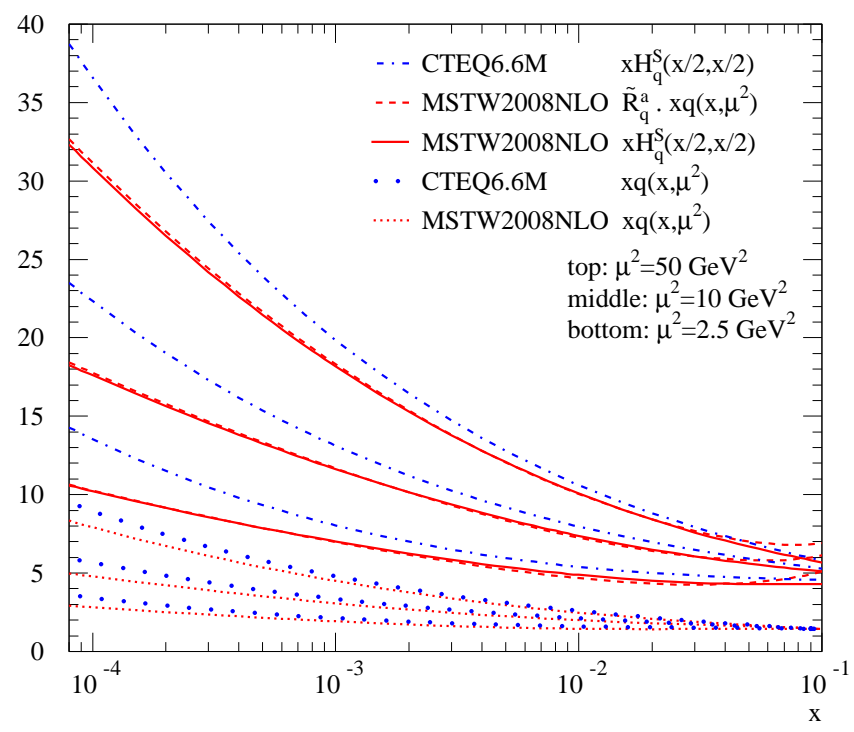

Figure 9: Skewed (solid) and diagonal (dotted lines) quark singlet distributions at scales $\mu^{2}=$ $2.5,10,50 \mathrm{GeV}^{2}$, for MSTW2008NLO and CTEQ6.6M as indicated in the legend. Shown as dashed lines (MSTW only) is the approximation using the analytic skewing factor $\tilde{R}^{a}$ from (17). 
for $x \gg \xi$ we can neglect the $\xi$ dependence. Secondly, we see a much larger skewing enhancement for the quarks than for the gluons. Indeed, it is known that, in the leading $\ln 1 / x$ approximation (LLA), the skewed distributions are equal to the diagonal ones [31]. As mentioned above, this arises because, in the LLA, the longitudinal fractions, $x$, of the momentum are strongly ordered. Already at the first evolution step we have $x \gg \xi$. Hence the $\xi$ dependence becomes negligible. Now for gluons, with spin 1 , the LLA correponds to a flat $x g(x)=$ constant distribution. With a parameterisation of the form $x g \sim x^{-\lambda}$, we see from Fig. 4 that for $\lambda>0(\lambda<0)$ the skewed distribution is enhanced (suppressed) in comparison with the diagonal gluon. On the other hand, for a pair of $t$-channel quarks with spin $\frac{1}{2}$, the LLA corresponds to a flat $q(x)=$ constant behaviour, see (3). That is, for the form $x q \sim x^{-\lambda}$, we have no skewed effect if $\lambda=-1$, but a large enhancement for small $\lambda$ close to zero. In the latter case, with $\lambda \gtrsim 0$, the structure of the loop integration is such that it prefers to transfer the major part of the momentum flow along one quark propagator.

This behaviour is demonstrated in Figs. 6and7, at the lowest scale. We see that the Shuvaev transform actually suppresses the gluon. This is expected when we look at the $x$ behaviour of the diagonal gluon; inspection of Fig. 4 shows that $\lambda_{g}$ becomes negative here. This is also demonstrated in the $\mu^{2}=2.5 \mathrm{GeV}^{2}$ curves in Fig. 8, For MSTW gluons, we have a suppression of the diagonal gluon, whereas for CTEQ gluons, there is no net effect at this particular choice of parameters. This behaviour is demonstrated with the lowest curve for $x \lesssim 3 \cdot 10^{-2}$ in the lower panel of Fig. 8; in this regime CTEQ partons have $\lambda_{g} \simeq 0$. As the scale increases, the transform enhances the diagonal gluons.

We emphasise that the use of the $x g, x q \propto x^{-\lambda}$ forms does not mean that we assume power-like asymptotics for $x \rightarrow 0$. Clearly, this oversimplified parameterisation can be valid only in a limited range of energy or $x$. We have used it here simply to qualitatively illustrate some of the main features of the skewed effect. Actually, in practice, and in the supplied grids, we determine the GPDs directly in terms of the well known diagonal distributions, which have much more complicated $x$ structure, using the full Shuvaev transforms, (15) and (6) .

\subsection{Leading order skewed distributions}

For completeness, we also calculate LO generalised parton distributions. The LO integrated distributions have a steeper $1 / x$ behaviour than those at NLO; that is, the values of $\lambda$ are larger. Therefore the skewed effect is larger. All other features of the LO generalised distributions are qualitatively the same as those at NLO, see Fig. 10 in comparison to Figs. 8 and 9 ,

The large numerical difference between the LO and NLO integrated gluon is due, first, to

the absence of the LO coefficient function for $\gamma^{*} g$ splitting, $C_{\gamma g}^{(0)}=0$; and, second, to a singular $1 / z$ term in the quark-quark splitting function $P_{q q}$ which is present at NLO, but absent at LO. To compensate for these absences at LO, we need to 'artificially' enhance the input LO gluon at low $x$. It is, therefore, not necessarily true that the LO contribution to another process can 

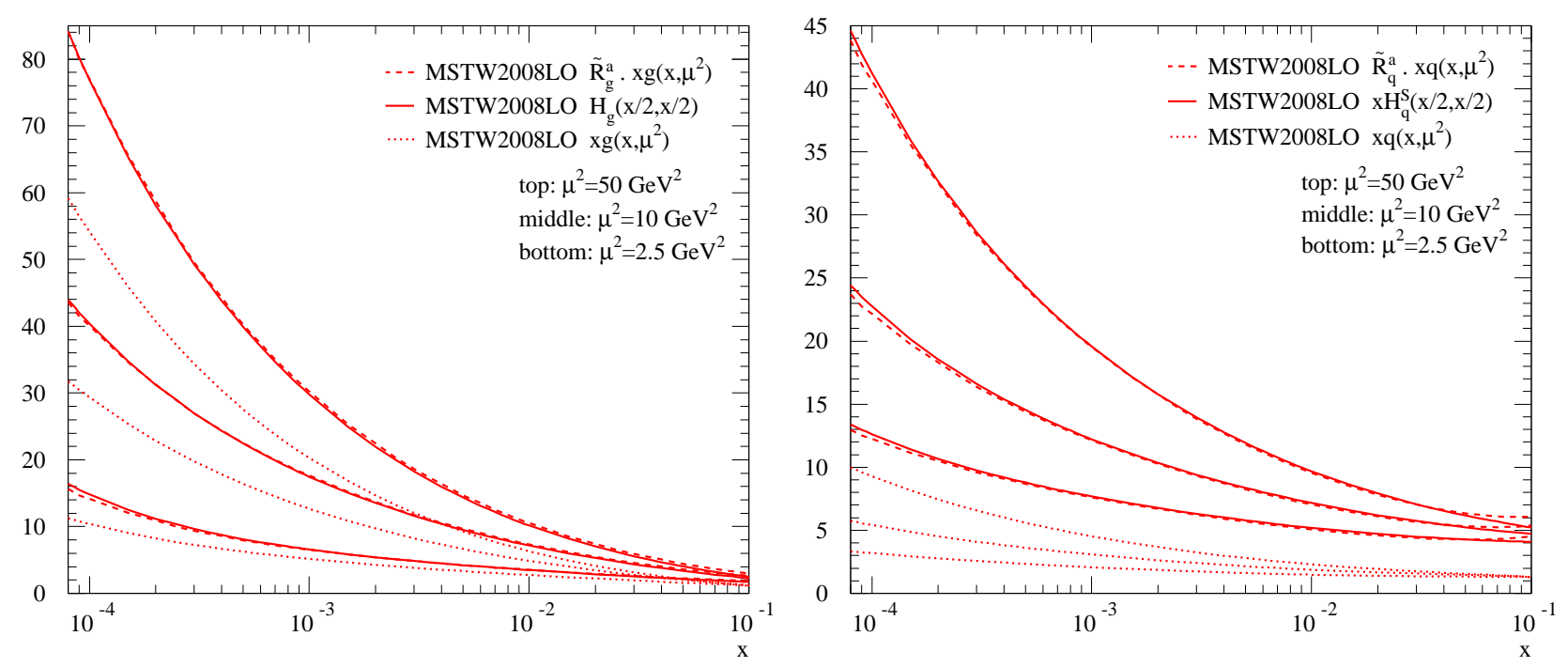

Figure 10: Skewed (solid and dashed) and diagonal (dotted lines) LO partons from MSTW2008 as a function of $x$ at scales $\mu^{2}=2.5,10,50 \mathrm{GeV}^{2}$. The dashed lines show the skewed partons if the analytic approximation $\tilde{R}^{a}$ from (17) is used. Left: gluons, right panel: $u, d, s$ quark singlet.

be effectively replaced by the same enhanced gluons. For this reason we prefer to use NLO partons.

Recall that the description of DVCS data in terms of the LO skewed distribution, given by the Shuvaev approach, was not good [32], while at NLO we observe agreement with the data 23]. The data have also been described in terms of a dipole model [25]. Here, the interaction of the gluon with the quark-antiquark pair produced by the photon plays the role of the NLO coefficient function $C_{\gamma g}$, while the skewed effect of the NLO gluon was calculated using the Shuvaev prescription. Therefore, actually, the dipole approach is close to a NLO treatment of DVCS.

\subsection{Grid interpolation package}

As the numerical computation of the full Shuvaev transform requires some care and can be too slow for applications, we provide a simple and fast interpolation routine in Fortran77. This includes reasonably small (approx. 3.4 MBytes) grid files for the LO and NLO input global analyses MSTW2008 [29], MRST2004 [33] and CTEQ6.6 [30]. These contain the information for quarks, antiquarks and gluons. The interpolation routine and grid files can be downloaded from http://www.maths.liv.ac.uk/TheorPhys/RESEARCH/pubcodes.html 4 The grid files use 85 points in $x$ where $4 \cdot 10^{-5} \leq x \leq 1$, together with 43 points in the ratio $\xi / x$ where $0 \leq \xi / x \leq 1$

\footnotetext{
${ }^{4}$ Grid files for other partons can be created upon request.
} 
and 20 points in $q^{2}$ where $1.25 \mathrm{GeV}^{2} \leq q^{2} \leq 80 \mathrm{GeV}^{2}$. Using $\xi / x$ as opposed to $\xi$ as a parameter increases the interpolation accuracy as the region $\xi \lesssim x$, where the skewed distributions are rising sharply, can be sampled more densely than regions $\xi \ll x$, where they are flatter. The $x$ grid points were chosen on a log scale, and the transform uses a linear interpolation on log scales for the three parameters (apart from the bin which includes $\xi / x=0$, where the interpolation is performed on a linear scale). The evolution in $q^{2}$ is very smooth, so fewer points in $q^{2}$ are needed.

The accuracy of the results from the interpolation on the grid is always better than $0.2 \%$ in the small $x$ regime (and still better than $1 \%$ for $x>10^{-2}$ ), and typically much better for $\xi \ll x$ or in the case of gluons. Based on convergence tolerances of the double integrals evaluated in (15. 6), and the numerical derivatives (of the interpolated input partons) of one per mille, we estimate the accuracy of the grid points to be better than $0.2 \%$ in the small $x$ regime.

Note that while in the figures above we have shown the skewed partons only up to $x=0.1$, the grid files contain information up to $x=1$. Of course at large $x$ and $\xi$ we cannot justify the results, while at small $\xi$ and large $x$ the skewed distributions are approaching the diagonal ones.

\section{Comparison with an alternative approach}

In general there are two possibilities to parameterise GPDs. One is to relate skewed distributions to the well known diagonal (global) parton distributions. This can be done with the help of the double distributions proposed by Radyushkin [20, 21]. The general form contains an arbitrary new function (up to the normalisation condition), but by construction it reduces to the diagonal distributions in the limit $\xi \rightarrow 0$. The Shuvaev transform is a particular case of this approach, with the advantage that after a physically reasonable assumption (that is, no singularities in the right-half $j$ plane) it gives unique GPDs in the low $x$ domain in terms of global diagonal partons, without any new parameters. The physical reasonableness of this assumption is evident from Fig. 2 and the accompanying discussion.

An alternative approach is to fit the available data, corresponding to both skewed and diagonal distributions, using an ansatz or model for the input GPDs at some starting scale $Q_{0}$. This approach was used in a recent paper [34], where the moments of the GPDs were parameterised in terms of beta $(B)$ functions motivated by an $\mathrm{SO}(3)$ partial wave model, see Section 3.2 of [34]. In order not to lose the statistical significance of the DVCS data, which corresponds to the skewed distributions, only a small subset of global $\left(F_{2}\right)$ data were included in the analysis. Moreover, we stress that the DVCS data do not cover the whole kinematic domain of $x$ and $\xi$, but rather correspond to the special situation $|x|=\xi$. It was emphasised in [34] that it is impossible to describe HERA data with a model with one leading SO(3) partial wave, especially at LO. Based on this observation, the authors claim that GPDs given by the Shuvaev transform are not applicable, that is, are in contradiction with the DVCS data. Note, 
however, that the integral corresponding to the Shuvaev transform is determined directly from global diagonal partons, which have a more complicated $x$ behaviour. It is well known that already diagonal 'global' data cannot be described by a single Regge pole ansatz. Moreover global diagonal partons are poorly described at LO [29]. The NLO corrections are large, since, in comparison with LO, they contain qualitatively new $1 / z$ singularities in the splitting and coefficient functions. Furthermore, note that the analytic formula for the ratid5 (15), which indeed is written for one Regge pole, was given just to qualitatively illustrate the discussion.

Going to NLO, we see no contradiction of the integral, parameter-free, Shuvaev transform approach with the available low $x$ DVCS data [25]. Moreover, the ratios $r$ of skewed-to-diagonal PDFs given in Fig. 7 of [34] for the NLO $\overline{\mathrm{MS}}$ scheme are very close to those that we obtain using the Shuvaev transform with recent global partons. Table 1 shows the agreement between the NLO $\overline{\mathrm{MS}}$ GPDs of [34] and our values obtained using the Shuvaev transform at $x=0.001$ for $Q^{2}=10$ and $50 \mathrm{GeV}^{2}$. It is clear from the Table that the difference between the GPDs based on the Shuvaev transform and those obtained in [34] by the NLO $\overline{\mathrm{MS}}$ fit to the 'skewed' DVCS data, is much less than the DVCS error bars.

\begin{tabular}{|c|c|c|c|c|}
\hline \multirow{2}{*}{} & \multicolumn{2}{|c|}{$Q^{2}=10 \mathrm{GeV}^{2}$} & \multicolumn{2}{c|}{$Q^{2}=50 \mathrm{GeV}^{2}$} \\
\cline { 2 - 5 } & $r_{G}$ & $r_{Q S}$ & $r_{G}$ & $r_{Q S}$ \\
\hline CTEQ6.6M & 1.04 & 1.67 & 1.06 & 1.71 \\
MSTW2008NLO & 1.03 & 1.65 & 1.05 & 1.70 \\
NLO MS GPDs of [34] & 1.06 & 1.76 & 1.05 & 1.77 \\
\hline
\end{tabular}

Table 1: Comparison of the skewed-to-diagonal ratio for gluons $r_{G}=H_{g}(x, x) / H_{g}(x, 0)$ and $u, d, s$ quark singlet $r_{Q S}=H_{q}^{S}(x, x) / x H_{q}^{S}(x, 0)$ at $x=0.001$ for scales $Q^{2}=10$ and $50 \mathrm{GeV}^{2}$. Diagonal CTEQ6.6M and MSTW2008NLO partons are used as input to calculate the skewed distributions, and the resulting ratios are compared to the ratios as displayed in Fig. 7 of [34] using their NLO $\overline{\mathrm{MS}}\left(\sum-\mathrm{PW}\right)$ GPDs.

\section{Conclusions}

We have shown how the skewed (or generalised) parton distributions, $H_{i}(x, \xi)$, that are needed to describe diffractive processes, can be obtained, for small $x$, directly from the conventional integrated global parton densities, $f_{i}(x)$. This parameter-free method is based on the Shuvaev transform, and is applicable to accuracy $O(\xi)$ at NLO, which should be sufficient for the description of all diffractive processes of interest. For the reasons given in the previous section the alternative approach of Ref. [34] does not have the advantage of being parameter free.

\footnotetext{
"It is misleading to quote the ratio as "conformal" (as was done for eq.(28) of 34]) since it relies on a single Regge pole ansatz, as well as on conformal symmetry.

${ }^{6}$ The accuracy is $O\left(\xi^{2}\right)$ at LO.
} 
First we identified the reason for the doubts which had been raised concerning the use of the Shuvaev transform. Further, we noted that the Shuvaev transform follows from the physicallymotivated conjecture that the small $x$ input is specified by Regge physics, and so does not generate any singularities in the right-half $(j>1)$ plane in the space-like $(x>\xi)$ domain. The transform therefore has practical applicability. We then investigated the kinematic range of reliability of the simplified analytic formula (15). This formula was derived for $x=\xi$, assuming that the unintegrated parton distributions $x f_{i}$ have a power-like $x^{-\lambda_{i}}$ behaviour. The 'analytic' formula was only introduced to illustrate some of the qualitative features of GPDs.

In order to obtain accurate generalised parton distributions at NLO at any small $x$, $\xi$, we performed a detailed computation of the integrals of the Shuvaev transform. Finally, these computations allow us to provide a readily accessible package which allows the evaluation of GPDs for arbitrary small $x, \xi$ in the space-like $(x>\xi)$ domain. These NLO GPDs should facilitate the theoretical description of all diffractive processes of interest.

\section{Appendix: The inverse transform}

Although we do not need the inverse transformation for the calculation of the skewed distributions for small $\xi$ from diagonal partons using the Shuvaev transform, it is still worth noting that the inverse problem is solvable. Here we shall briefly clarify the inverse transformation and show that the regions $|x|>|\xi|$ and $|x|<|\xi|$ transform separately.

Our aim is to find the function $f(x)$ whose Mellin moments,

$$
M_{N}=\int \frac{\mathrm{d} x}{x} x^{N} f(x)
$$

are equal to Gegenbauer moments of the GPD $H(x, \xi)$,

$$
G_{N}(\xi)=\frac{N ! \Gamma(3 / 2)}{2^{N} \Gamma(N+3 / 2)} \int \mathrm{d} x \xi^{N} C_{N}^{\frac{3}{2}}(x / \xi) H(x, \xi),
$$

$G_{N}(\xi)=M_{N}$. Here for definiteness we consider the non-singlet channel, the singlet one can be treated in the same manner.

With the generating function for Gegenbauer polynomials,

$$
\begin{aligned}
\xi^{N} C_{N}^{\frac{3}{2}}(x / \xi) & =\left.\frac{1}{N !} \frac{\partial^{N}}{\partial t^{N}} R^{-\frac{3}{2}}(t, x)\right|_{t=0}, \\
R(t, x) & =1-2 t x+t^{2} \xi^{2},
\end{aligned}
$$

the moments take the form

$$
G_{N}(\xi)=\frac{1}{2} \int_{0}^{1} \mathrm{~d} y \int_{0}^{1} \mathrm{~d} v(1-v)^{-\frac{1}{2}} \frac{1}{2 \pi i} \oint \frac{d t}{t^{N+1}} R^{-\frac{3}{2}}\left(\frac{1}{2} v t, y\right) H(y, \xi),
$$


where the contour of the $t$-integration goes in the complex plane around $t=0$, while the integral over $v$ yields the normalisation. Integrating by parts over $y$ (this is needed to make the kernel less singular) we rewrite this expression as

$$
\begin{aligned}
G_{N}(\xi)= & -\int_{0}^{1} \mathrm{~d} y \int_{0}^{1} \frac{d v}{v}(1-v)^{-\frac{1}{2}} \frac{1}{2 \pi i} \oint \frac{d t}{t^{N+1}} \frac{1}{t} R^{-\frac{1}{2}}\left(\frac{1}{2} v t, y\right) \frac{\partial}{\partial y} H(y, \xi) \\
& +\left.\int_{0}^{1} \frac{\mathrm{d} v}{v}(1-v)^{-\frac{1}{2}} \frac{1}{2 \pi i} \oint \frac{d t}{t^{N+1}} \frac{1}{t} R^{-\frac{1}{2}}\left(\frac{1}{2} v t, y\right) H(y, \xi)\right|_{-1} ^{1} .
\end{aligned}
$$

The contour of $t$-integration can be shrunk to the cut of the radical in the integrand,

$$
\begin{aligned}
-\frac{1}{2 \pi i} \oint \frac{d t}{t^{N+1}} \frac{1}{t} R^{-\frac{1}{2}}\left(\frac{1}{2} v t, y\right) & =\frac{1}{\pi} \int_{t_{2}}^{t_{1}} \frac{\mathrm{d} t}{t^{N+1}} \frac{1}{t} R_{c}^{-\frac{1}{2}}(t, y) \\
& =-\frac{1}{\pi} \int_{\frac{1}{t_{2}}}^{\frac{1}{t_{1}}} \frac{\mathrm{d} x}{x} x^{N+1} R_{c}^{-\frac{1}{2}}\left(\frac{1}{x}, y\right),
\end{aligned}
$$

where the values $t_{1,2}=2 /\left(\xi^{2} v\right)\left[y \pm \sqrt{y^{2}-\xi^{2}}\right]$ determine the real (for $|y|>|\xi|$ ) interval, in which

$$
R_{c}(t, y)=v y t-\frac{1}{4} \xi^{2} v^{2} t^{2}-1>0 .
$$

These relations show that the desired function is given by the integral transformation

$$
f(x)=\int_{0}^{1} \mathrm{~d} y K(x, y)\left[\frac{\partial}{\partial y}+\delta(y-1)-\delta(y+1)\right] H(y, \xi),
$$

with the kernel

$$
\begin{gathered}
K(x, y)=-\frac{1}{\pi} \int_{0}^{1} \frac{\mathrm{d} v}{v}(1-v)^{-\frac{1}{2}}\left[x^{2} Y^{-\frac{1}{2}} \theta(Y)\right], \\
Y \equiv v x y-\frac{1}{4} \xi^{2} v^{2}-x^{2}, \quad|y|>|\xi| .
\end{gathered}
$$

Note that for $|\xi| \ll 1$ the transformation becomes close to the identity, $f(x)=H(x)+\mathcal{O}(\xi)$.

If $|y|<|\xi|$ the branch points $t_{1,2}$ are complex. In this case the function $f$ will be defined on the segment lying in the complex plane. Thus the inverse transform does not exist as a real function for the whole interval.

\section{Acknowledgements}

We thank Markus Diehl, Dieter Müller and Graeme Watt for valuable discussions, and Graeme Watt for the provision of the MSTW2008 partons prior to publication. 


\section{References}

[1] A. D. Martin, M. G. Ryskin and T. Teubner, Phys. Rev. D 62 (2000) 014022.

[2] A. D. Martin, M. G. Ryskin and T. Teubner, Phys. Lett. B 454 (1999) 339.

[3] M. Diehl and W. Kugler, Eur. Phys. J. C 52 (2007) 933.

[4] S. V. Goloskokov and P. Kroll, Eur. Phys. J. C 53 (2008) 367.

[5] V. A. Khoze, A. D. Martin and M. G. Ryskin, Phys. Lett. B 401 (1997) 330; Eur. Phys. J. C 14 (2000) 525; ibid. C 23 (2002) 311.

[6] A. D. Martin, C. Nockles, M. G. Ryskin and T. Teubner, Phys. Lett. B 662 (2008) 252.

[7] M. Diehl and D. Y. Ivanov, Eur. Phys. J. C 52 (2007) 919.

[8] T. Ohrndorf, Nucl. Phys. B 198 (1982) 26.

[9] A. P. Bukhvostov, G. V. Frolov, L. N. Lipatov and E. A. Kuraev, Nucl. Phys. B 258 (1985) 601.

[10] S. J. Brodsky, P. Damgaard, Y. Frishman and G. P. Lepage, Phys. Rev. D 33 (1986) 1881.

[11] D. Müller, Phys. Rev. D 49 (1994) 2525.

[12] X. D. Ji, J. Phys. G 24 (1998) 1181.

[13] X. D. Ji, Phys. Rev. Lett. 78 (1997) 610.

[14] X. D. Ji, Phys. Rev. D 55 (1997) 7114.

[15] A. Shuvaev, Phys. Rev. D 60 (1999) 116005.

[16] A. V. Belitsky, D. Müller, L. Niedermeier and A. Schäfer, Nucl. Phys. B 546 (1999) 279.

[17] A. V. Belitsky and D. Müller, Nucl. Phys. B 537 (1999) 397.

[18] M. Diehl and W. Kugler, Phys. Lett. B 660 (2008) 202.

[19] A. V. Radyushkin, Phys. Rev. D 56 (1997) 5524.

[20] A. V. Radyushkin, Phys. Rev. D 59 (1999) 014030.

[21] A. V. Radyushkin, Phys. Lett. B 449 (1999) 81.

[22] I. V. Musatov and A. V. Radyushkin, Phys. Rev. D 61 (2000) 074027.

[23] K. Kumericki, D. Müller and K. Passek-Kumericki, Nucl. Phys. B 794 (2008) 244. 
[24] J. D. Noritzsch, Phys. Rev. D 62 (2000) 054015.

[25] L. Favart, M. V. T. Machado and L. Schoeffel, Braz. J. Phys. 37 (2007) 798; see also arXiv:hep-ph/0511069.

[26] D. Müller and A. Schäfer, Nucl. Phys. B 739 (2006) 1.

[27] M. V. Polyakov and A. G. Shuvaev, arXiv:hep-ph/0207153.

[28] A. G. Shuvaev, K. J. Golec-Biernat, A. D. Martin and M. G. Ryskin, Phys. Rev. D 60 (1999) 014015.

[29] A. D. Martin, W. J. Stirling, R. S. Thorne and G. Watt, arXiv:0901.0002.

[30] CTEQ Collaboration, P. M. Nadolsky et al., Phys. Rev. D 78 (2008) 013004.

[31] J. Bartels and M. Loewe, Z. Phys. C 12 (1982) 263.

[32] K. Kumericki, D. Müller and K. Passek-Kumericki, arXiv:0807.0159 (hep-ph).

[33] A. D. Martin, R. G. Roberts, W. J. Stirling and R. S. Thorne, Phys. Lett. B 604 (2004) 61.

[34] K. Kumericki and D. Müller, arXiv:0904.0458 (hep-ph). 